\title{
Revealing the Dynamics of Functional Nanomaterials in Their Formation and Application Media with Liquid and Gas-phase TEM
}

Damien Alloyeau ${ }^{1}$, Abdelali Khelfa ${ }^{1}$, Kinan Aliyah ${ }^{2}$, Adrian Chmielewski ${ }^{3}$, Jun Meng ${ }^{4}$, Hakim Amara ${ }^{5}$, Hazar Guesmi ${ }^{4}$, Jaysen Nelayah ${ }^{1}$, Guillaume Wang ${ }^{1}$, Cyrille Hamon ${ }^{2}$, Doru Constantin ${ }^{2}$ and Christian Ricolleau $^{1}$

${ }^{1}$ CNRS - University of Paris / Laboratoire Matériaux et phénomènes Quantiques, Paris, Ile-de-France, France, ${ }^{2}$ CNRS - Paris Saclay University / Laboratoire de physique du solide, Orsay, Ile-de-France, France, ${ }^{3}$ Pennsylvania State University, University Park, Pennsylvania, United States, ${ }^{4}$ Institut Charles Gerhardt, Montpellier, Languedoc-Roussillon, France, ${ }^{5}$ ONERA / University of Paris, Paris, Ile-deFrance, France

The development of efficient nanotechnologies requires fabricating functional nanomaterials with controlled morphology, composition and atomic structure, and determining if and how these structural parameters change under working conditions. Therefore, understanding the dynamics of nanostructures in their formation and application media is a crucial challenge for materials science. In that regards, in situ TEM in closed cells provides the unique opportunity to visualize at the appropriated scale the behavior of nanomaterials in well controlled liquid and gaseous environments.

We have exploited liquid-cell TEM to study the growth and etching mechanisms of plasmonic nanostructures ( $\mathrm{Au}$ and $\mathrm{AuAg}$ nanorods). We will show that this in situ technique allows identifying the individual role of chemicals in the reaction media, helping our understanding of nanomaterial synthesis. We will notably demonstrate the key role of capping agents in anisotropic growth and selective etching processes which illustrate the necessity to consider the hybrid nature (i.e. organic/inorganic) of functional nanomaterials to unravel their formation mechanisms.[1,2]

We have also used gas-cell TEM to identify the nature of the active sites for $\mathrm{O}_{2}$ and $\mathrm{H}_{2}$ adsorption/dissociation on gold nanoparticles supported on $\mathrm{TiO}_{2}$. Our real-space observations of gold catalysts under "realistic" conditions (i.e. atmospheric pressure and high temperature) provide a clear picture of their structural evolution in functional state which sheds a new light on the facet-specific molecular adsorption of $\mathrm{O}_{2 \cdot[3]}$

So far, the lack of statistical results and the questioning about the effects of the beam on nanomaterials dynamics have limited the impact of in situ TEM investigations in the fields of colloidal chemistry and heterogeneous catalysis. These intrinsic limitations have pushed us to corroborate all our TEM results with complementary in situ techniques (SAXS and absorption spectroscopy) or atomistic simulations to enhance the validity of our conclusions. Such correlative approaches are essential to allay the skepticism about in situ TEM results and maximize their impact in materials science. 
a
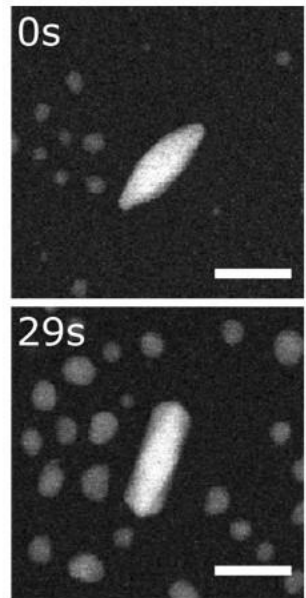
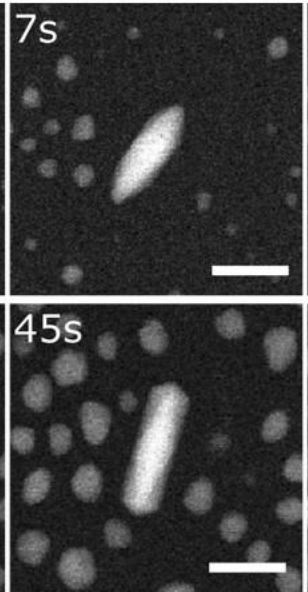
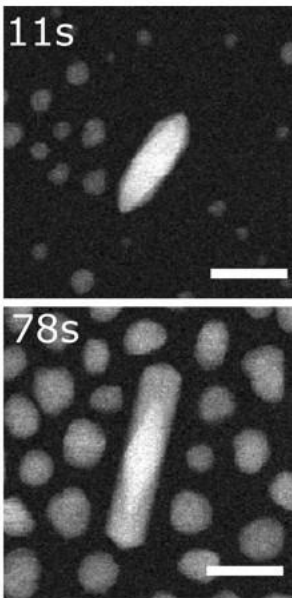

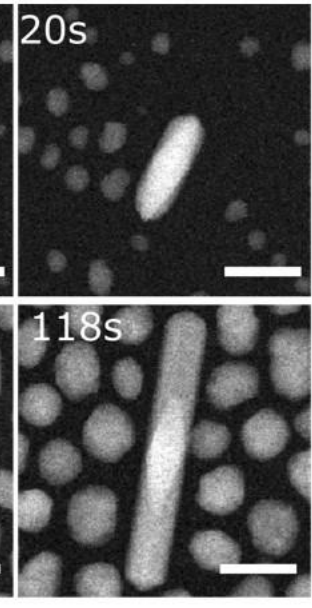

Figure 1. Figure 1. (a) Time-lapse STEM images showing the anisotropic deposition of silver on a gold bipyramid in aqueous media. Scale bar is $50 \mathrm{~nm}$ on all images. (b) Contour plots of the AuAg rods extracted every second from image analysis. Extracted with permission from ref. [2].

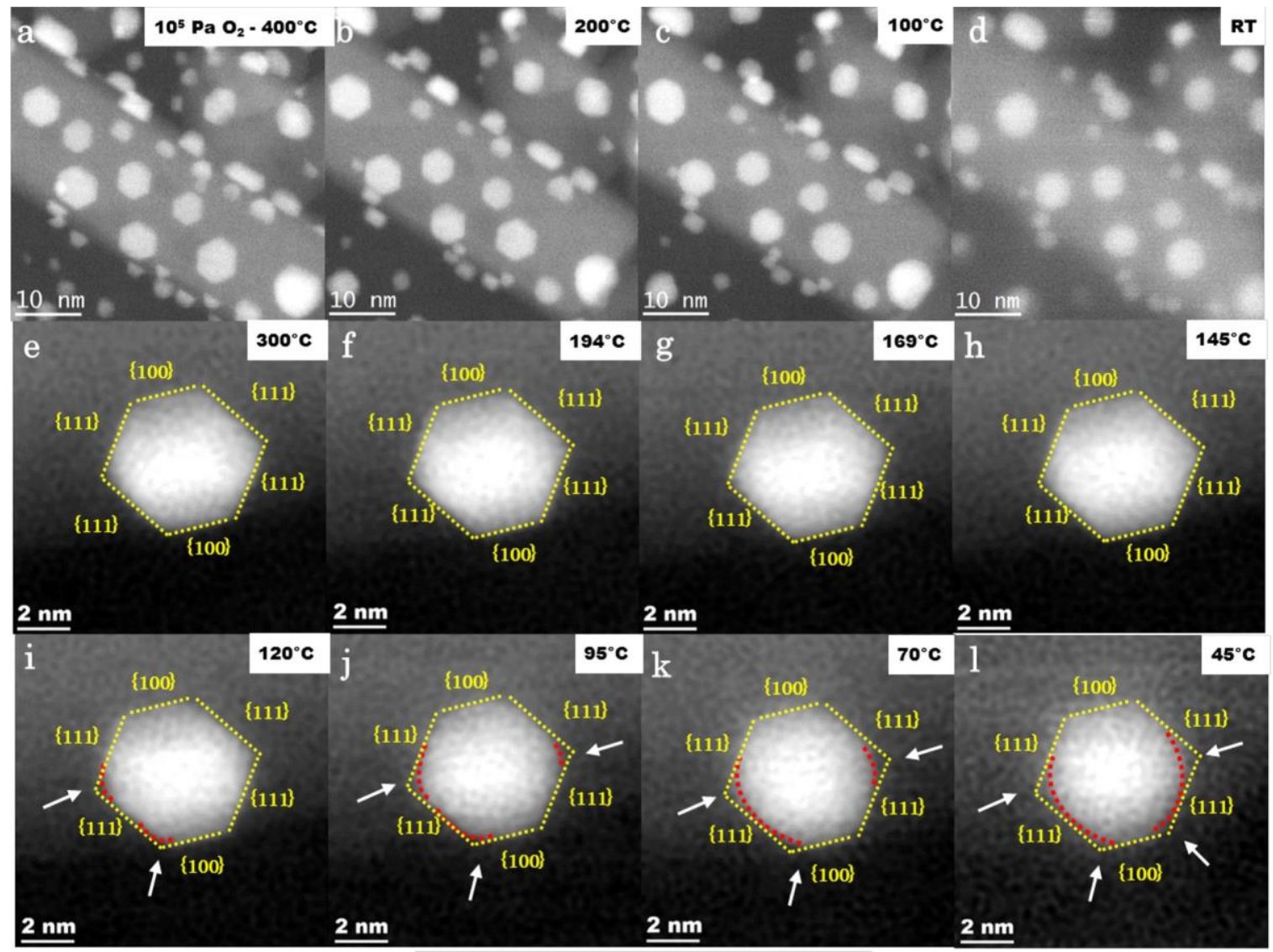

Figure 2. In situ HAADF-STEM observations of Au nanoparticles on r-TiO2 (110) surfaces under O2 at atmospheric pressure. (a-d) Temperature series of low magnification HAADF-STEM images showing that rounding if Au nanoparticles at low temperature. (e-1) Temperature series of high magnification HAADFSTEM images over the temperature window where the octahedron-to-round morphology transition is observed. This shape transition is due to the growth of (110) facets on which $\mathrm{O} 2$ molecules preferentially react. Extracted with permission from ref. [3]. 


\section{References}

[1] Ahmad et al. Nanoletters, 17 (2017), pp. 4194

[2] Aliyah et al. ChemRxiv (2019) DOI : ACS Nano 13 (2019), pp. 2024 